\title{
High-temperature interface superconductivity in bilayer copper oxide films by pulsed laser deposition
}

\author{
Jia-hao Deng ${ }^{1 \dagger}$, Tian-shuang Ren ${ }^{1 \dagger}$, Le-le Ju${ }^{1}$, Hong-rui Zhang ${ }^{2}$, Ji-rong Sun ${ }^{2}$, Bao-gen Shen ${ }^{2}$ and \\ Yan-wu Xie $\mathrm{Xi}^{1, *}$
}

\begin{abstract}
In a seminal work, Gozar et al. reported on the high-temperature interface superconductivity in bilayers of insulating $\mathrm{La}_{2} \mathrm{CuO}_{4}$ and metallic $\mathrm{La}_{2-x} \mathrm{Sr}_{x} \mathrm{CuO}_{4}(x=0.45)$. An interesting question to address is how general and robust this interface superconductivity is. In the past, the cuprate bilayers were grown in a unique atomic-layer molecular beam epitaxy system, with a Sr doping range of $x \leq 0.47$, and the atomically flat interface was thought to be indispensable. Here, we have fabricated bilayers of $\mathrm{La}_{2} \mathrm{CuO}_{4}$ and $\mathrm{La}_{2-x} \mathrm{Sr}_{x} \mathrm{CuO}_{4}$ by pulsed laser deposition. We have tried to extend the nominal doping range of $\mathrm{Sr}$ from the previous maximum of 0.47 to the present 1.70 (the nominal Sr content in the targets). X-ray diffraction result indicates that our $\mathrm{La}_{2-x} \mathrm{Sr}_{x} \mathrm{CuO}_{4}$ films with $x \leq 0.60$ have very high crystalline quality; but the film crystalline structure degrades gradually with further increasing $x$, and finally the structure is fully lost when $x$ reaches 1.40 and higher. Although the film quality scatters dramatically, our experiments show that there exists superconductivity for bilayers in nearly the entire over-doped $\mathrm{Sr}$ range, except for a non-superconducting region at $x \sim 0.80$. These observations demonstrate that the interface superconductivity in copper oxides is very general and robust.
\end{abstract}

Keywords: cuprate, superconductivity, interface, pulsed laser deposition, high temperature superconductivity

\section{INTRODUCTION}

The effect of interface on superconductivity has received much attention. It was demonstrated that superconductivity can occur at the interface of two nonsuperconducting materials [1-6], and the critical temperature $\left(T_{c}\right)$ can even be significantly enhanced com- pared with the corresponding single-phase samples [1,79]. One of the most intriguing interfaces is the bilayer of $\mathrm{La}_{2} \mathrm{CuO}_{4}$ and $\mathrm{La}_{2-x} \mathrm{Sr}_{x} \mathrm{CuO}_{4}[\mathrm{LSCO}(x)]$ films. In a seminal work, Gozar et al. [1] reported on the existence of high- $T_{\mathrm{c}}$ superconductivity with an enhanced $T_{c}$ at the interface between insulating $\mathrm{La}_{2} \mathrm{CuO}_{4}$ and metallic $\mathrm{La}_{1.55} \mathrm{Sr}_{0.45} \mathrm{CuO}_{4}$. A following work showed that the high- $T_{\mathrm{c}}$ superconductivity could reside in a single $\mathrm{CuO}_{2}$ plane on the $\mathrm{La}_{2} \mathrm{CuO}_{4}$ side [7]. The $\mathrm{Sr}$ interdiffusion across the interface was restricted [1], and the superconductivity was attributed mainly to the interface electronic redistribution [10]. An anomalous independence of interface superconductivity from carrier density was observed [11]. These discoveries make the bilayer of $\mathrm{La}_{2} \mathrm{CuO}_{4}$ and $\operatorname{LSCO}(x)$ a base for studying the origin of high-temperature superconductivity and interface emergent phenomena [12].

$\operatorname{LSCO}(x)$ is the simplest superconducting cuprate system that has single $\mathrm{CuO}_{2}$ conducting layers whose hole concentration is determined by the $\mathrm{Sr}$ and/or oxygen concentration. It is insulating for $x<0.06$, superconducting for $0.06 \leq x \leq 0.30$, metallic for $0.30<x<0.80$, and insulating again for $x \geq 0.80[13,14]$. Previous studies on bilayers of $\mathrm{La}_{2} \mathrm{CuO}_{4}$ and $\operatorname{LSCO}(x)$ showed that the bilayers sustained superconductivity when the $\operatorname{LSCO}(x)$ layer was superconducting $(0.06 \leq x \leq 0.30)$ [11,15], and created interface superconductivity when the $\operatorname{LSCO}(x)$ layer was metallic [1], with a maximum $x$ of 0.47 [11]. Will the interface superconductivity persist with further increasing $x$, particularly for $x \geq 0.80$ where the $\operatorname{LSCO}(x)$ layer becomes insulating again? Unfortunately, the answer to it is unknown because the wide doping range for

\footnotetext{
${ }^{1}$ Interdisciplinary Center of Quantum Information, Zhejiang Province Key Laboratory of Quantum Technology and Device, and Department of Physics, Zhejiang University, Hangzhou 310027, China

2 Beijing National Laboratory for Condensed Matter Physics \& Institute of Physics, Chinese Academy of Sciences, Beijing 100190, China

${ }^{3}$ Collaborative Innovation Center of Advanced Microstructures, Nanjing University, Nanjing 210093, China

$\uparrow$ These authors contribute equally to this work.

* Corresponding author (email: ywxie@zju.edu.cn)
} 
$x>0.47$ is unexplored yet. The lacking of relevant studies might be in part because the heavily Sr-riched $\operatorname{LSCO}(x)$ films are not stable due to oxygen vacancies $[13,16]$. In previous studies $[1,5-7,11]$ of interface superconductivity, the bilayers were grown in a unique atomic-layer-by-layer molecular beam epitaxy (MBE) system that was fantastic in making atomically flat high-quality samples, but might not be suitable in making the heavily Sr-riched $\operatorname{LSCO}(x)$ films. In contrast, due to its thermodynamically nonequilibrium nature, pulsed laser deposition (PLD) can be used to produce metastable films far from chemical equilibrium [17]. In this work, using PLD, we extensively explore the interface superconductivity of the bilayers of $\mathrm{La}_{2} \mathrm{CuO}_{4}$ and $\mathrm{LSCO}(x)$, with the nominal doping level $x$ spanning from 0.00 to 1.70 (the nominal $\mathrm{Sr}$ content in the PLD targets). Our result shows that, although the film quality scatters, the high- $T_{\mathrm{c}}$ interface superconductivity is very general and can be achieved in a wide range of $\mathrm{Sr}$ doping levels.

\section{EXPERIMENTAL SECTION}

\section{Target fabrication}

The $\operatorname{LSCO}(x)$ targets for PLD growth were prepared by conventional solid-state reaction using $\mathrm{La}_{2} \mathrm{O}_{3}$ (99.9\%), $\mathrm{SrCO}_{3}$ (99.95\%), and $\mathrm{CuO}$ (99\%) powders. These powders were weighted with a ratio of the nominal composition of $\operatorname{LSCO}(x)$, mixed, and pre-heated at $900^{\circ} \mathrm{C}$ (except $1050^{\circ} \mathrm{C}$ for $\mathrm{La}_{2} \mathrm{CuO}_{4}$ ) for $24 \mathrm{~h}$ in air. After that, they were pressed into 1 inch-diameter pellets and sintered at $1100^{\circ} \mathrm{C}$ for $24 \mathrm{~h}$ or longer in air.

\section{Film growth}

All the films were grown on (001) $\mathrm{LaSrAlO}_{4}$ single-crystalline substrates. Before growth, the substrates were preannealed in situ at $800^{\circ} \mathrm{C}$ under $1 \times 10^{-4}$ mbar oxygen $\left(\mathrm{O}_{2}\right)$ for $20 \mathrm{~min}$. The films grew at $730^{\circ} \mathrm{C}$, under a mixed gas atmosphere of 0.033 mbar nitrous oxide $\left(\mathrm{N}_{2} \mathrm{O}\right)$ and 0.077 mbar $\mathrm{O}_{2}$, followed by an in-situ post-annealing at $550^{\circ} \mathrm{C}$, under $200 \mathrm{mbar} \mathrm{O}_{2}$, for $1 \mathrm{~h}$, and then were cooled to room temperature under the same gas condition. A $\mathrm{KrF}$ excimer laser $(248 \mathrm{~nm})$ was used. The laser fluence was $0.95 \mathrm{~J} \mathrm{~cm}^{-2}$. The laser spot size on target was $3.0 \mathrm{~mm}^{2}$. The repetition rate was $2 \mathrm{~Hz}$. The sample-target distance was $55 \mathrm{~mm}$. The nominal $x$ for all the $\operatorname{LSCO}(x)$ films simply refers to $x$ in the corresponding $\operatorname{LSCO}(x)$ targets.

\section{Thickness control}

The film thickness was determined by counting the growth laser pulses. The growth rate was about $1 \mathrm{~nm}$ per
100 pulses, which was calibrated by measuring low-angle $\mathrm{X}$-ray reflectance (XRR) on a few $\operatorname{LSCO}(x)$ single-phase films (e.g., Supplementary information, Fig. S1). We deposited all the $\operatorname{LSCO}(x)$ single films with 4000 pulses, and all the bilayers with 4000 pulses for the bottom layer and 1000 pulses for the top layer.

\section{Transport measurement}

The typical sample size was $3.0 \mathrm{~mm}$ in length and $2.0 \mathrm{~mm}$ in width. Four electrodes $(0.5 \mathrm{~mm}$ in length, and $2.0 \mathrm{~mm}$ in width) distributed evenly along the long side by evaporating silver on the top of the single-phase and bilayer films. These electrodes were connected by $\mathrm{Al}$ wire bonder, and used to measure the samples' resistances in a conventional four-probe method.

\section{$\mathrm{X}$-ray characterization}

The X-ray diffraction (XRD) was performed using $\mathrm{Cu} \mathrm{Ka}$ radiation $(\lambda=1.5406 \AA)$ on a Bruker AXS D8-Discover.

\section{RESULTS}

\section{Crystalline structure of single $\operatorname{LSCO}(x)$ films}

We have grown single and bilayer films with nominal composition $\mathrm{LSCO}(x)$ on (001) $\mathrm{LaSrAlO}_{4}$ single-crystalline substrates by PLD. First, we characterized the structure of the single $\operatorname{LSCO}(x)$ films by XRD. Fig. 1 shows XRD data of the samples. The presence of the characteristic $\mathrm{K}_{2} \mathrm{NiF}_{4}$ structural peaks of $\left(\begin{array}{lll}0 & 2 n\end{array}\right)$ indicates epitaxial growth of the films on the $\mathrm{LaSrAlO}_{4}$ substrates. A close look of the XRD data shows clear finite-thickness oscillations around the film peaks for samples of $0.00 \leq x \leq 0.60$ (see Fig. 2), indicating the high quality of the films $[14,18]$ in this range. However, peaks from impurity phase were observed for $x=0.80$ and 0.90 (see Fig. 1). The $\left(\begin{array}{lll}0 & 0 & 2 n\end{array}\right)$ film peaks are visible until the $\mathrm{Sr}$ content reaches $x=1.10$, but become invisible for $x \geq 1.40$, indicating a full loss of epitaxial growth (see Fig. 1). We point out that although $\operatorname{LSCO}(x)$ has attracted huge research interests for years, very few reports [13] have been released on the heavily overdoped $\operatorname{LSCO}(x)$, partly because of the instability of the structure due to the loss of oxygen [19]. Heavily overdoped $\operatorname{LSCO}(x)$ films of reasonable quality were only reported by reactive coevaporation for $0.00 \leq x \leq 2.00$ [13], and by oxide molecular beam epitaxy for $0.00 \leq x \leq 1.00$ [14]. The $x$ dependence of the $c$-axis lattice parameter calculated from the data shown in Fig. 1 (see Fig. 3) is consistent with the corresponding results in the two previous studies $[13,14]$, implying that our film quality in the whole $x$ range is 


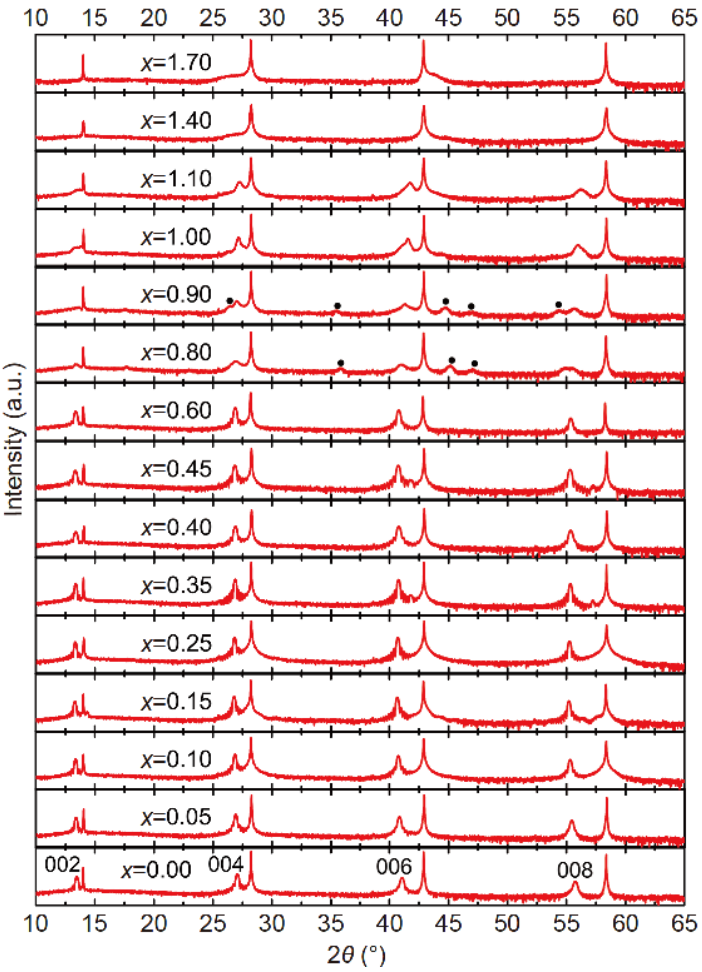

Figure $1 \mathrm{XRD}$ of the $\operatorname{LSCO}(x)$ thin films with different nominal $\mathrm{Sr}$ contents $(x)$. Peak indices are shown at the bottom and correspond to both film (the left) and $\mathrm{LaSrAlO}_{4}$ substrate (the right) peaks. The black dots for $x=0.80$ and 0.90 indicate peaks from unspecified impurity phase. No epitaxial peaks were observed for $x=1.40$ and 1.70 , indicating a full loss of epitaxial film structure. The thickness for all the films is around $40 \mathrm{~nm}$.

reasonable. Atomic force microscopy (AFM) images (see Fig. S2) show that the surface morphology is relatively smooth when $x$ is small but becomes rough for the heavily overdoped samples. In short, these data confirm that high-quality $\operatorname{LSCO}(x)$ films can be obtained by PLD for $0.00 \leq x \leq 0.60$, while the film quality gradually degrades with further increasing $x(0.80 \leq x \leq 1.10)$, and finally the epitaxial crystalline structure is fully lost for $x \geq 1.40$. Here we caution that the nominal $x$ for $x \geq 1.40$ is immaterial because the films are likely not in a singlephase. The use of $x$ in that range is only for simplicity.

\section{Transport properties of single $\operatorname{LSCO}(x)$ films}

As shown in Fig. 4, the undoped $\mathrm{La}_{2} \mathrm{CuO}_{4}$ film $(x=0.00)$ shows a highly insulating behavior. The $x=0.05$ film still shows an insulating behavior but its overall resistance is much lower than that of $x=0.00$ film. Superconducting behavior was found for the $x=0.10,0.15$, and 0.25 samples, and the $x=0.15$ sample has the best superconducting properties. Further increase in doping level changes the

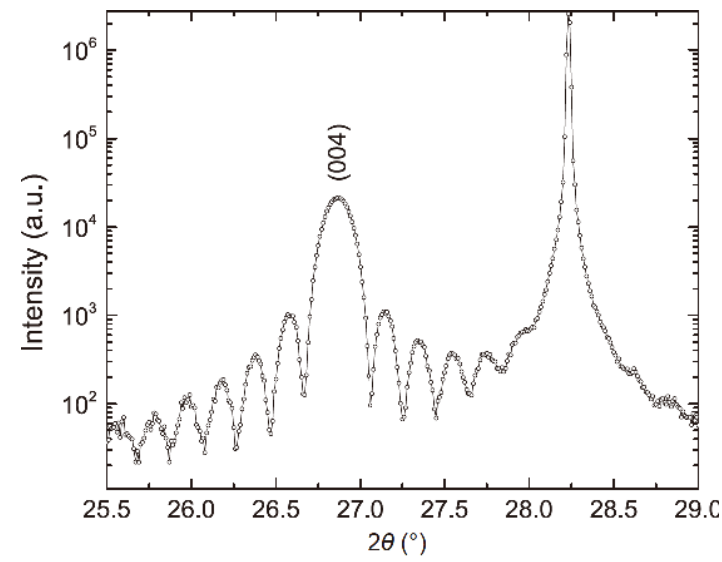

Figure 2 Enlarged view of the XRD data of the $\operatorname{LSCO}(0.35)$ film shown in Fig. 1 demonstrates clear finite-thickness oscillations in the vicinity of the $\left(\begin{array}{lll}0 & 0 & 4\end{array}\right)$ reflection, confirming the high-quality of the film. Similar oscillations were observed for the $\operatorname{LSCO}(x)$ films with $x$ varying from 0.00 to 0.60 .

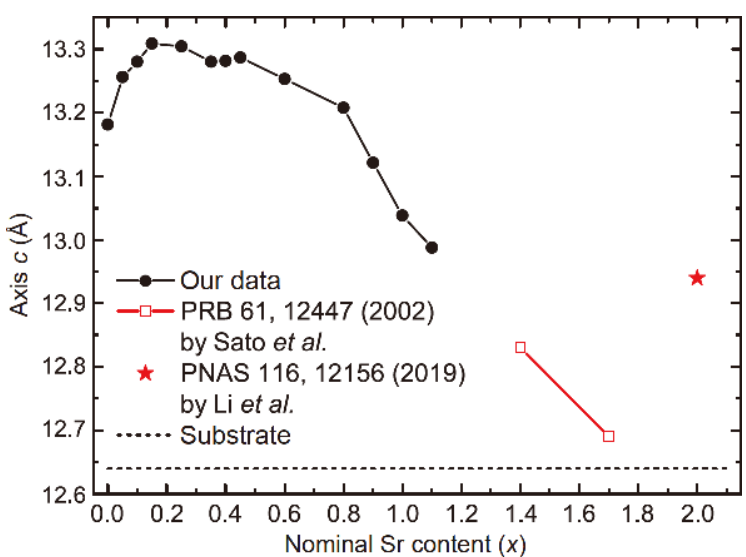

Figure 3 Out-of-plane $c$-axis lattice parameter of films as a function of nominal Sr content $(x)$. Derived from the XRD data shown in Fig. 1. The $x=1.40,1.70$, and 2.00 data are regenerated from previous studies as noted. The dashed line indicates the axis $c$ value of (001) $\mathrm{LaSrAlO}_{4}$ substrate.

transport into a metallic behavior $(0.35 \leq x \leq 0.60$, inset of Fig. 4). These behaviors agree well with the well-known $\operatorname{LSCO}(x)$ phase diagram, implying that the real Sr content of our samples is very close to the nominal $x$ value. With further increasing $x$ to above 0.80 , however, the films become insulating again, and the overall resistance increases with $x$ until $x=1.10$. For $x=1.40$ and 1.70 , the films are still highly insulating but show a weaker temperature dependence that might be attributed to the collapse of their crystalline structures. Overall, the single $\operatorname{LSCO}(x)$ films prepared by PLD show comparable transport properties with the films prepared by reactive coeva- 


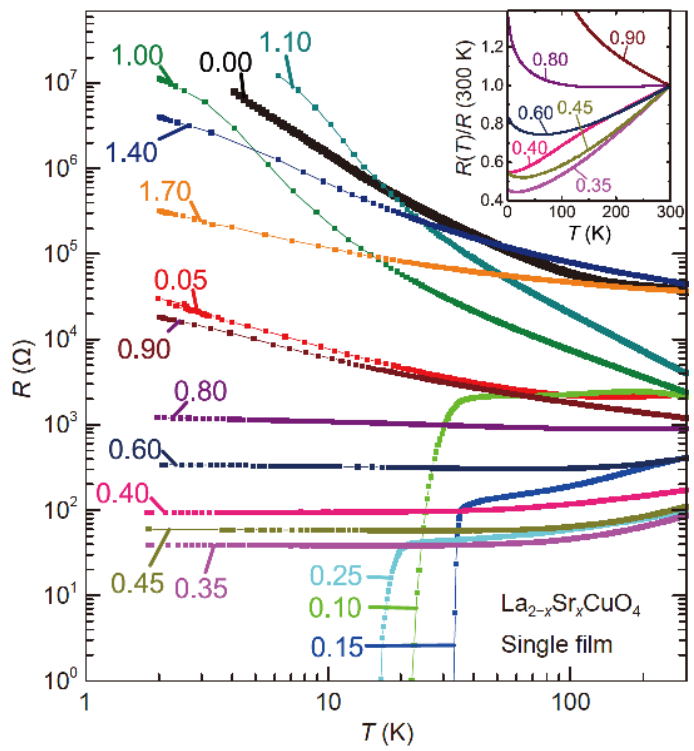

Figure 4 The dependence of resistance on temperature for single films of different nominal Sr contentns $(x)$. The Sr content $x$ of each film is as labeled in the figure. The top inset shows partial data in linear coordinate, in order to better identify their transport behaviors. The undoped $\mathrm{La}_{2} \mathrm{CuO}_{4}(x=0.00)$ is shown in thicker black symbols. The thickness for all the films is around $40 \mathrm{~nm}$. Double logarithmic coordinates are used for clarity.

poration [13] or oxide molecular beam epitaxy [14].

\section{Transport properties of bilayers}

Previously, Gozar et al. [1] showed that the interface between the insulating $(x=0.00)$ and the metallic $(x=0.45)$ $\operatorname{LSCO}(x)$ films was superconducting with high $T_{c}$, and the deposition sequence mattered. Here we first show that this seminal work can be well reproduced by PLD deposition, as demonstrated in Fig. 5. In order to explore thoroughly the possible interface superconductivity, we further extensively combined $\mathrm{La}_{2} \mathrm{CuO}_{4}$ and $\mathrm{LSCO}(x)$, for
$0.05 \leq x \leq 1.70$, with both sequences. Fig. 6 shows the temperature-dependent resistance of bilayers with $\mathrm{La}_{2} \mathrm{CuO}_{4}$ on the bottom [hereafter labeled as $\mathrm{La}_{2} \mathrm{CuO}_{4}+$ $\operatorname{LSCO}(x)$ ]; Fig. 7 shows the temperature-dependent resistance of bilayers with $\operatorname{LSCO}(x)$ on the bottom [hereafter labeled as $\operatorname{LSCO}(x)+\mathrm{La}_{2} \mathrm{CuO}_{4}$ ]. In each sequence of bilayers, the thicknesses of the bottom layer and the top layer are around 40 and $10 \mathrm{~nm}$, respectively. The most important observation is that the bilayers exhibit superconductivity for a very wide $x$ range.

The bilayers of both sequences are still insulating for $x=0.05$, and superconducting for $0.10 \leq x \leq 0.25$, similar to the single $\operatorname{LSCO}(x)$ films. For $x \geq 0.35$, the overdoped range, while the single $\operatorname{LSCO}(x)$ films are either metallic $(0.35 \leq x \leq 0.60)$ or insulating $(x \geq 0.80)$, the bilayers of both sequences are nearly always superconducting, except for an abnormality around $x=0.80 \quad(x=0.60$ and 0.80 for $\mathrm{La}_{2} \mathrm{CuO}_{4}+\mathrm{LSCO}(x)$, Fig. 6 ; $x=0.80$ and 0.90 for $\operatorname{LSCO}(x)+$ $\mathrm{La}_{2} \mathrm{CuO}_{4}$, Fig. 7). Therefore, our present experiments indicate that the interface superconductivity is very general in the bilayers of $\mathrm{LSCO}(x)$ and $\mathrm{La}_{2} \mathrm{CuO}_{4}$. It is quite striking to see that, although the bilayers are of poor crystalline structure for $x \geq 1.40$ (see Fig. 1 and Fig. S2), they still exhibit good superconducting behaviors, suggesting that the interface superconductivity is a very robust phenomenon and is insensitive to the interface quality. Similar observation was also found recently in a $\mathrm{Ba}_{0.8} \mathrm{Sr}_{0.2} \mathrm{TiO}_{3} / \mathrm{La}_{2} \mathrm{CuO}_{4}$ heterointerface [20].

To compare different samples in a more objective way, we define $T_{\mathrm{c}}$ by the maximal slope of $R(T)$ that can be determined unambiguously as the temperature of the maximum of the derivative curve, $\mathrm{d} R(T) / \mathrm{d} T$ [11]. In Fig. 8, based on the transport data shown in Figs 6 and 7, and the $T_{\mathrm{c}}$ derived thereafter, we constructed phase diagrams for the bilayers of both sequence. "S", "M", and "I" represent the superconducting, metallic, and insulating
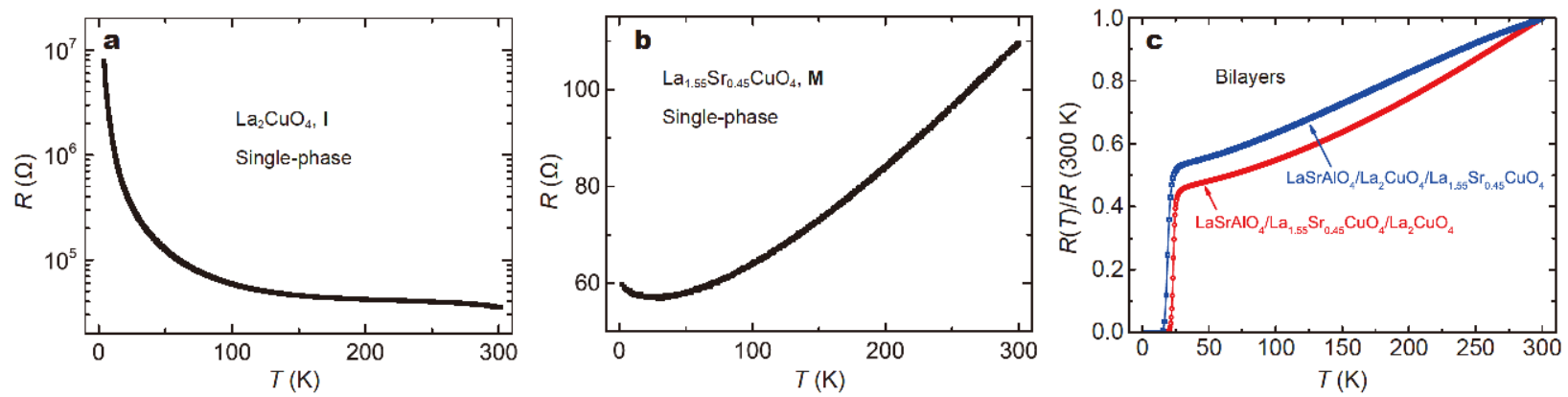

Figure 5 The dependence of resistance on temperature for single and bilayer films for $x=0.45$. (a) $\mathrm{La}_{2} \mathrm{CuO}_{4},(\mathrm{~b}) \mathrm{La}_{1.55} \mathrm{Sr}_{0.45} \mathrm{CuO}_{4}$, and (c) the bilayer of $\mathrm{La}_{2} \mathrm{CuO}_{4}$ and $\mathrm{La}_{1.55} \mathrm{Sr}_{0.45} \mathrm{CuO}_{4}$. The $\mathrm{LaSrAlO}_{4}$ is the substrate. In each sequence the thicknesses of the bottom layer and top layer are around 40 and $10 \mathrm{~nm}$, respectively. 


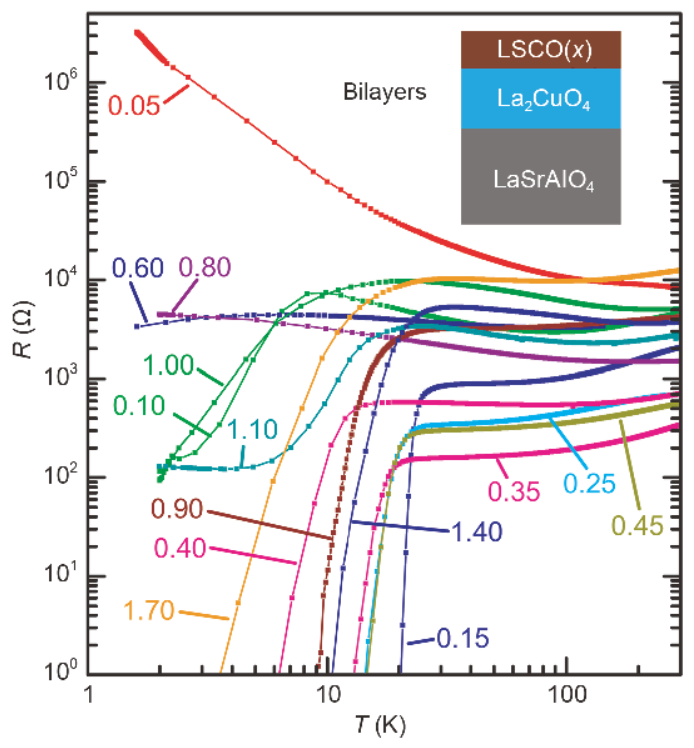

Figure 6 The dependence of resistance on temperature for $\mathrm{La}_{2} \mathrm{CuO}_{4}+$ $\operatorname{LSCO}(x)$ bilayers. The nominal Sr content $x$ of each sample is as labeled in the figure. The top inset shows the schematic of the bilayers. The thicknesses of $\mathrm{La}_{2} \mathrm{CuO}_{4}$ and $\mathrm{LSCO}(x)$ films are around 40 and $10 \mathrm{~nm}$, respectively. Double logarithmic coordinates are used for clarity.

regions, respectively. "T" represents the region where the transport behavior involves an insulating-to-metallic or metallic-to-insulating transition (see Fig. S3 for an example). The $T_{\mathrm{c}}$ of single $\operatorname{LSCO}(x)$ films (small circles and dashed line) and the bilayer data from the previous studies of Bozovic group [1,11] (the red stars and dots) are also included for comparison. Firstly, one can see that, for each sequence, the $T_{\mathrm{c}}$ for bilayers of high $x$ value is comparable to that of low $x$ value. This result agrees with the previous observation that the $T_{c}$ of interface superconductivity is less dependent on the doping level of $x$ [11]. Secondly, consistent with the previous study on the bilayers of $x=0.45$ [1], for almost all the $x$ values, the interface superconductivity depends on the sequence, and the overall $T_{\mathrm{c}}$ of $\mathrm{LSCO}(x)+\mathrm{La}_{2} \mathrm{CuO}_{4}$ is higher than that of the $\mathrm{La}_{2} \mathrm{CuO}_{4}+\mathrm{LSCO}(x)$ [1]. The appearance of these two features suggests that the superconductivity observed in the bilayers of the present study is likely of the same nature as that in the previous studies [1,10,21]. We note that the $T_{\mathrm{c}}$ obtained in our study is comparable or slightly inferior to that of the as-grown bilayers from Bozovic's group (the red empty stars in Fig. 8), but much less than that of the bilayers that had been optimally annealed in ozone (the red closed star and the red dotted line in Fig. 8b). This difference might originate from oxygen stoichiometry because all the samples used in our studies had not been treated by ozone. In addition, as shown in

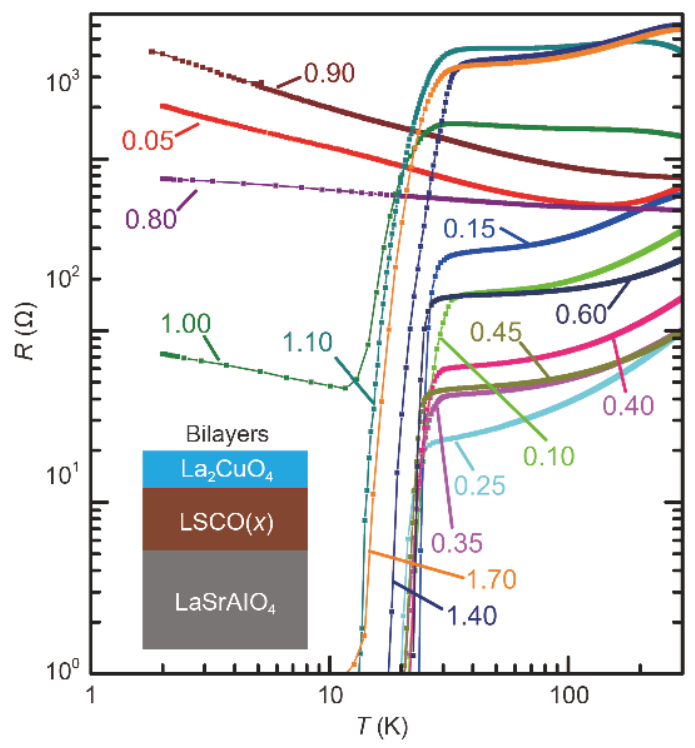

Figure 7 The dependence of resistance on temperature for $\operatorname{LSCO}(x)+$ $\mathrm{La}_{2} \mathrm{CuO}_{4}$ bilayers. The nominal Sr content $x$ of each sample is as labeled in the figure. The bottom inset shows the schematic of the bilayers. The thicknesses of $\operatorname{LSCO}(x)$ and $\mathrm{La}_{2} \mathrm{CuO}_{4}$ films are around 40 and $10 \mathrm{~nm}$, respectively. Double logarithmic coordinates are used for clarity.

Figs 4 and 8 (the circles and dashed line), even for the single-phase $\operatorname{LSCO}(x)$, the $T_{\mathrm{c}}$ is lower compared with that grown by oxide MBE, indicating that the film quality of samples prepared by PLD is somehow lower than that of samples prepared by oxide MBE.

\section{DISCUSSION}

Previously, two mechanisms, cation interdiffusion and electronic redistribution, have been considered as the possible causes for the high- $T_{\mathrm{c}}$ interface superconductivity, and the electronic redistribution has been suggested to be the dominant one $[1,6,10]$. Similarly, our present observations can be explained better by electronic redistribution rather than cation interdiffusion. If cation interdiffusion is the dominant mechanism, one would expect that the bilayers for $x$ around 0.80 are superconducting since there is no simple reason that the interdiffusion of Sr should drop at this particular content. In the electronic redistribution scenario, the mobile charge carriers (holes) are depleted from the overdoped $\operatorname{LSCO}(x)$ and accumulated in $\mathrm{La}_{2} \mathrm{CuO}_{4}$, according to their chemical potential difference. The chemical potential in $\operatorname{LSCO}(x)$ inferred from X-ray photoemission data decreases with increasing $x$ [22]. This explains the interface electronic redistribution. Unfortunately, the chemical potential data are not available for $x>0.30$. If it continues to decrease with increasing $x$ in the full range up to 1.70 , 

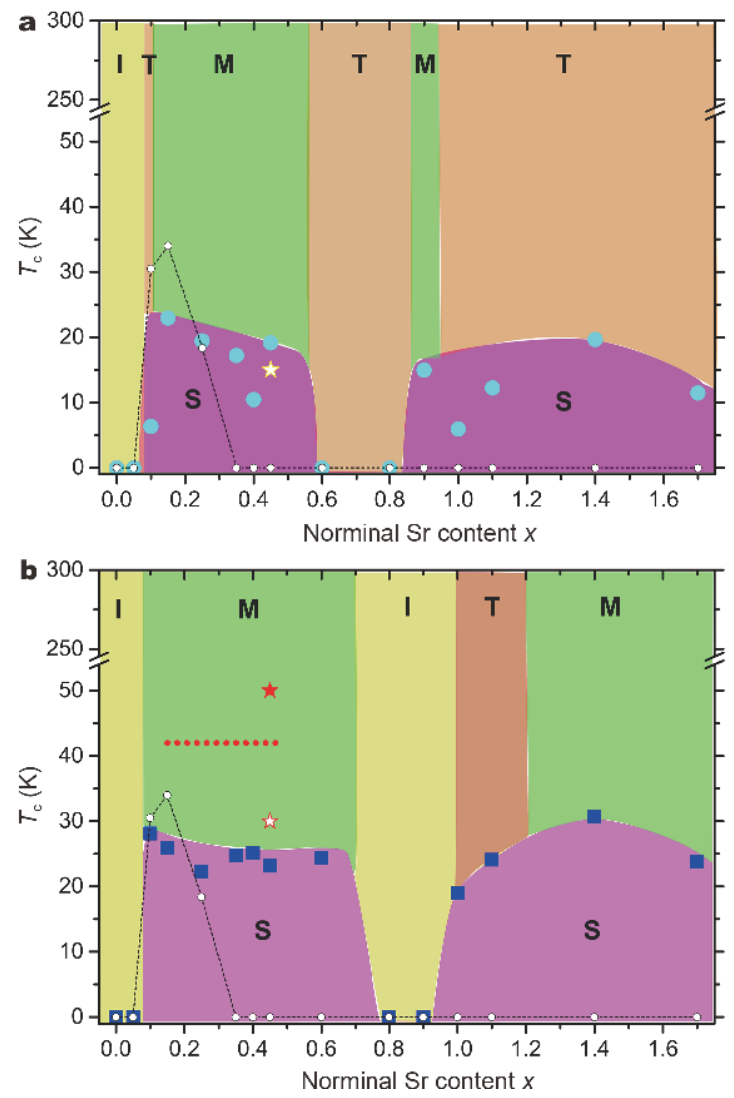

Figure 8 Phase diagrams for (a) $\mathrm{La}_{2} \mathrm{CuO}_{4}+\mathrm{LSCO}(x)$ and (b) $\mathrm{LSCO}(x)+$ $\mathrm{La}_{2} \mathrm{CuO}_{4}$ bilayers, derived from the data shown in Figs 6 and 7. $T_{\mathrm{c}}$ is defined as the transition onset that is determined as the position of the maximum of the derivative curve, $\mathrm{d} R(T) / \mathrm{d} T$. The cyan circles in (a) and the blue squares in (b) represent our experimental data. The empty stars in (a) and (b) represent the corresponding bilayers reported by Bozovic's group [1]; the red closed star in (b) represents the maximum $T_{\mathrm{c}}$ reached by Bozovic's group [1] by thoroughly annealing the bilayer in ozone. The red dotted line in (b) represents the Sr-doping range that has been studied by Bozovic's group [11] and the corresponding $T_{\mathrm{c}}$. S, M, I and T denote superconducting, metallic, insulating, and transitional between metallic and insulating, respectively. $\mathbf{T}$ is exampled in Fig. S3. For comparison, the $T_{\mathrm{c}} v$ s. $x$ of the single $\operatorname{LSCO}(x)$ films is shown in both panels with small circles and dashed line.

one would again expect that all the bilayers for $x>0.30$ are superconducting due to electronic redistribution, inconsistent with the experimental data. This inconsistence might be reconciled by considering the issue of oxygen non-stoichiometry. It is well known that $\operatorname{LSCO}(x)$ tends to lose oxygen with increasing $x$ [18]. So, the effect of increasing $x$ is two-fold: while the $\mathrm{Sr}$ substitution for La can dope holes into the $\mathrm{CuO}_{2}$ planes and decrease the chemical potential, the increased oxygen vacancies will reduce the amount of holes and increase the chemical potential. In this consideration, the abnormality around $x=0.80$, might be explained by that in this region the effect of oxygen vacancies surpasses the effect of doping holes. This interpretation is partially supported by that around $x=0.80$, the $R(T)$ curve of $\operatorname{LSCO}(x)$ changes dramatically from metallic to insulating (inset of Fig. 4). An alternative explanation is that the electronic redistribution is induced by oxygen diffusion [23].

Although electronic redistribution is a sound mechanism, it is still quite interesting and puzzling that, for $x \geq 1.00$, the bilayers of both sequences become superconducting again. In this case, the interface superconductivity is actually formed between two insulating copper oxides. One possibility is that in this range the effect of doping holes is stronger than the effect of oxygen vacancies, and thus the corresponding $\operatorname{LSCO}(x)$ layer has a low chemical potential that causes electronic redistribution. Another possibility is that there are inhomogeneous impurity phases that are of low chemical potential, and thus can transfer holes to $\mathrm{La}_{2} \mathrm{CuO}_{4}$. In addition, because of the poor crystalline structure in the high $x$ range, the corresponding bilayers might have much severer cation interdiffusion than that observed in the previous studies [1,7]. At present, we are unable to distinguish all these possibilities yet. However, no matter what is the real underneath mechanism, it is clearly that the interface superconductivity is very general and not restricted to perfect crystalline structure. This point could be important for practical applications of interface superconductivity because it lowers the threshold to fabricate such devices.

Finally, we comment that there is actually one more intriguing possibility for the superconducting phase observed in the high $x$ range, that is, the superconductivity in the heavily over-doped cuprate. A few recent experimental [24,25] and theoretical [26,27] studies demonstrated that exotic superconductivity can exist in heavily overdoped cuprates. One case is the nodeless pairing in superconducting $\mathrm{CuO}_{2}$ monolayer grown on $\mathrm{Ba}_{2} \mathrm{Sr}_{2} \mathrm{CaCu}_{2} \mathrm{O}_{8+\delta}[24,26]$. Another case is the superconductivity in $\mathrm{Ba}_{2} \mathrm{CuO}_{4-y}$ in which the octahedron was found in an exceptionally compressed version $[25,27]$. Interestingly, as shown in Fig. 3, the $c$-axis lattice constant of $\operatorname{LSCO}(x)$ indeed decreases with increasing $x$ in the heavily overdoped range. Its value becomes close to that of $\mathrm{Ba}_{2} \mathrm{CuO}_{4-y}$ (the red star in Fig. 3) for $x>1.0$, suggesting that in this range the octahedral might be compressed as well. Further studies are needed to examine this possibility.

\section{CONCLUSIONS}

In summary, we have demonstrated for the first time the 
high- $T_{\mathrm{c}}$ interface superconductivity in the bilayers of $\mathrm{La}_{2} \mathrm{CuO}_{4}$ and overdoped $\operatorname{LSCO}(x)$ by PLD. The nominal Sr content $x$ has been significantly extended from the previous studies. Our result demonstrates that the superconductivity at this interface is robust and general. Since PLD is versatile in growing a large number of materials with reasonable quality, it provides the opportunity to combine more materials efficiently and thus may serve as a good tool to search for superconductivity in new interfaces.

\section{Received 24 July 2019; accepted 26 July 2019; published online 6 August 2019}

1 Gozar A, Logvenov G, Kourkoutis LF, et al. High-temperature interface superconductivity between metallic and insulating copper oxides. Nature, 2008, 455: 782-785

2 Reyren N, Thiel S, Caviglia AD, et al. Superconducting interfaces between insulating oxides. Science, 2007, 317: 1196-1199

3 Salvato M, Tieri G, Balestrino G, et al. Anisotropic properties of a single superconducting $\mathrm{CaCuO}_{2} / \mathrm{SrTiO}_{3}$ interface. Supercond Sci Technol, 2015, 28: 095012

4 Meir B, Gorol S, Kopp T, et al. Observation of two-dimensional superconductivity in bilayers of $\mathrm{BaBiO}_{3}$ and $\mathrm{BaPbO}_{3}$. Phys Rev B, 2017, 96: 100507

5 Baiutti F, Gregori G, Suyolcu YE, et al. High-temperature superconductivity at the lanthanum cuprate/lanthanum-strontium nickelate interface. Nanoscale, 2018, 10: 8712-8720

6 Suyolcu YE, Wang Y, Baiutti F, et al. Dopant size effects on novel functionalities: High-temperature interfacial superconductivity. Sci Rep, 2017, 7: 453

7 Logvenov G, Gozar A, Bozovic I. High-temperature superconductivity in a single copper-oxygen plane. Science, 2009, 326: 699-702

8 Koren G, Millo O. Enhancement of the superconducting transition temperature of $\mathrm{La}_{2-x} \mathrm{Sr}_{x} \mathrm{CuO}_{4}$ and $\mathrm{La}_{1.875} \mathrm{Ba}_{0.125} \mathrm{CuO}_{4}$ bilayers: $\mathrm{Bi}$ layer and reference film prepared on the same wafer. Phys Rev B, 2010, 81: 134516

9 Wang QY, Li Z, Zhang WH, et al. Interface-induced high-temperature superconductivity in single unit-cell FeSe films on $\mathrm{SrTiO}_{3}$. Chin Phys Lett, 2012, 29: 037402

10 Smadici S, Lee JCT, Wang S, et al. Superconducting transition at $38 \mathrm{~K}$ in insulating-overdoped $\mathrm{La}_{2} \mathrm{CuO}_{4}-\mathrm{La}_{1.64} \mathrm{Sr}_{0.36} \mathrm{CuO}_{4}$ superlattices: evidence for interface electronic redistribution from resonant soft X-ray scattering. Phys Rev Lett, 2009, 102: 107004

$11 \mathrm{Wu}$ J, Pelleg O, Logvenov G, et al. Anomalous independence of interface superconductivity from carrier density. Nat Mater, 2013, 12: $877-881$

12 Hwang HY, Iwasa Y, Kawasaki M, et al. Emergent phenomena at oxide interfaces. Nat Mater, 2012, 11: 103-113

13 Sato $\mathrm{H}$, Tsukada $\mathrm{A}$, Naito $\mathrm{M}$, et al. $\mathrm{La}_{2-x} \mathrm{Sr}_{x} \mathrm{CuO}_{y}$ epitaxial thin films $(x=0$ to 2$)$ : Structure, strain, and superconductivity. Phys Rev B, 2000, 61: 12447-12456

14 Johnson AM, Burquest FJ, Larsen HM, et al. Stabilization of Srrich ultrathin epitaxial films of $\mathrm{La}_{2-x} \mathrm{Sr}_{x} \mathrm{CuO}_{4}$. Thin Solid Films, 2018, 649: 167-170
15 Sen K, Marsik P, Das S, et al. Superconductivity and charge-carrier localization in ultrathin $\mathrm{La}_{1.85} \mathrm{Sr}_{0.15} \mathrm{CuO}_{4} / \mathrm{La}_{2} \mathrm{CuO}_{4}$ bilayers. Phys Rev B, 2017, 95: 214506

16 Torrance JB, Tokura Y, Nazzal AI, et al. Anomalous disappearance of high- $T_{\mathrm{c}}$ superconductivity at high hole concentration in metallic $\mathrm{La}_{2-x} \mathrm{Sr}_{x} \mathrm{CuO}_{4}$. Phys Rev Lett, 1988, 61: 1127-1130

17 Willmott PR, Huber JR. Pulsed laser vaporization and deposition. Rev Mod Phys, 2000, 72: 315-328

18 Bozovic I, Logvenov G, Belca I, et al. Epitaxial strain and superconductivity in $\mathrm{La}_{2-x} \mathrm{Sr}_{x} \mathrm{CuO}_{4}$ thin films. Phys Rev Lett, 2002, 89: 107001

19 Uchida S, Ido T, Takagi H, et al. Optical spectra of $\mathrm{La}_{2-x} \mathrm{Sr}_{x} \mathrm{CuO}_{4}$ : Effect of carrier doping on the electronic structure of the $\mathrm{CuO}_{2}$ plane. Phys Rev B, 1991, 43: 7942-7954

20 Pavlov DP, Zagidullin RR, Mukhortov VM, et al. Fabrication of high-temperature quasi-two-dimensional superconductors at the interface of a ferroelectric $\mathrm{Ba}_{0.8} \mathrm{Sr}_{0.2} \mathrm{TiO}_{3}$ film and an insulating parent compound of $\mathrm{La}_{2} \mathrm{CuO}_{4}$. Phys Rev Lett, 2019, 122: 237001

$21 \mathrm{Wu}$ J, Bollinger AT, He X, et al. Spontaneous breaking of rotational symmetry in copper oxide superconductors. Nature, 2017, 547: $432-435$

22 Ino A, Mizokawa T, Fujimori A, et al. Chemical potential shift in overdoped and underdoped $\mathrm{La}_{2-x} \mathrm{Sr}_{x} \mathrm{CuO}_{4}$. Phys Rev Lett, 1997, 79: 2101-2104

23 Jin $\mathrm{K}$, Bach $\mathrm{P}$, Zhang $\mathrm{XH}$, et al. Anomalous enhancement of the superconducting transition temperature of electron-doped $\mathrm{La}_{2-x} \mathrm{Ce}_{x} \mathrm{CuO}_{4}$ and $\mathrm{Pr}_{2-x} \mathrm{Ce}_{x} \mathrm{CuO}_{4}$ cuprate heterostructures. Phys Rev B, 2011, 83: 060511

24 Zhong $\mathrm{Y}$, Wang $\mathrm{Y}$, Han $\mathrm{S}$, et al. Nodeless pairing in superconducting copper-oxide monolayer films on $\mathrm{Bi}_{2} \mathrm{Sr}_{2} \mathrm{CaCu}_{2} \mathrm{O}_{8+\delta}$. Sci Bull, 2016, 61: 1239-1247

25 Li WM, Zhao JF, Cao LP, et al. Superconductivity in a unique type of copper oxide. Proc Natl Acad Sci USA, 2019, 116: 12156-12160

26 Jiang $\mathrm{K}, \mathrm{Wu} \mathrm{X}, \mathrm{Hu} \mathrm{J}$, et al. Nodeless high- $T_{\mathrm{c}}$ superconductivity in the highly overdoped $\mathrm{CuO}_{2}$ monolayer. Phys Rev Lett, 2018, 121: 227002

27 Liu K, Lu ZY, Xiang T. Electronic structures of quasi-onedimensional cuprate superconductors $\mathrm{Ba}_{2} \mathrm{CuO}_{3+\delta}$. Phys Rev Mater, 2019, 3: 044802

Acknowledgements This work was supported by the National Key Research and Development Program of Ministry of Science and Technology of China (2017YFA0303002, 2016YFA0300204, and 2016YFA0300701), and the Fundamental Research Funds for the Central Universities.

Author contributions Deng JH, Ren TS, and Ju LL performed the sample fabrication, transport measurements, and AFM characterization; Zhang HR, Sun JR, and Shen BG performed XRD measurements; Xie YW designed the experiment and wrote the manuscript. All authors contributed to data analysis and discussion.

Conflict of interest The authors declare that they have no conflict of interest.

Supplementary information The data of XRR and AFM, and an example for defining " $T$ ", are available in the online version of the paper. 


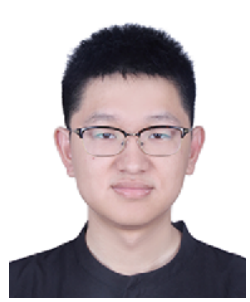

Jia-hao Deng is currently a master's degree candidate at the Department of Physics, Zhejiang University. He received his bachelor degree from Nanchang University in 2017. His research is focused on the interface superconductivity in $\mathrm{La}_{2-x} \mathrm{Sr}_{x} \mathrm{CuO}_{4}$.

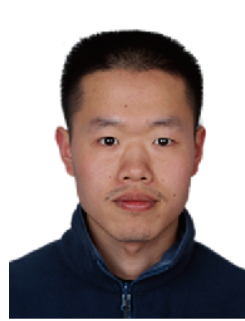

Tian-shuang Ren is currently a $\mathrm{PhD}$ candidate at the Department of Physics, Zhejiang University. He received his bachelor degree (majored in physics) from Lanzhou University in 2017. His PhD research focuses on the growth and characterization of iridates and high- $T_{\mathrm{c}}$ cuprates.

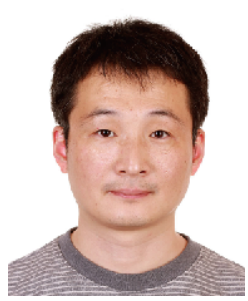

Yan-wu Xie is currently a professor at the Department of Physics, Zhejiang University. $\mathrm{He}$ received his $\mathrm{PhD}$ in physics from the Institute of Physics, CAS, in 2007. From 2007 to 2009, he was an associate professor at Yanshan University. From 2009 to 2011, he was a JSPS postdoctoral fellow at Tokyo University. From 2011 to 2015, he was a postdoctoral scholar and later a staff research associate at Stanford University. He joined Zhejiang University in June 2015. His research interests include superconductivity and electron gas at interfaces of complex oxides.
脉冲激光沉积法制备双层结构铜氧化物界面高温 超导

邓佳豪 ${ }^{1 \dagger}$, 任天爽 ${ }^{1 \dagger}$, 居乐乐 ${ }^{1}, 弓$ 洪瑞 $^{2}$, 孙继荣 ${ }^{2}$, 沈保根 $^{2}$, 谢燕武 ${ }^{1,3 *}$

摘要 在 2008 年发表的一篇经典工作中, Gozar等人报道了由绝缘 性的 $\mathrm{La}_{2} \mathrm{CuO}_{4}$ 和金属性的 $\mathrm{La}_{1.55} \mathrm{Sr}_{0.45} \mathrm{CuO}_{4}$ 双层薄膜构成的体系存在 界面超导. 一个重要的有待回答的问题是该界面超导是否强健以 及具有普遍性. 在Gozar等人的工作中, 铜氧化物双层结构是利用 特制的氧化物分子束外延设备生长的, 最大 $\mathrm{Sr}$ 掺杂量仅为 0.47 . 在 本工作中, 我们首次利用脉冲激光沉积法制备了铜氧化物双层结 构, 并重复出了上述界面超导工作. 在此基础上, 我们将 $\mathrm{Sr}$ 的掺杂 范围大幅扩展到 1.70 , 结果表明在由 $\mathrm{La}_{2} \mathrm{CuO}_{4}$ 和过掺杂的 $\mathrm{La}_{2-x} \mathrm{Sr}_{x} \mathrm{CuO}_{4}$ 构成的双层结构中界面超导非常强健和普遍. 值得一 提的是, 我们发现在 $x>0.8$ 范围内存在一个新的界面超导区间. 